\title{
A population pharmacokinetic model of cabozantinib in healthy volunteers and patients with various cancer types
}

\author{
Steven Lacy ${ }^{1}$ (1) $\cdot$ Bei Yang ${ }^{2} \cdot$ Jace Nielsen ${ }^{2} \cdot$ Dale Miles $^{1} \cdot$ Linh Nguyen $^{1} \cdot$ Matt Hutmacher $^{2}$
}

Received: 7 November 2017 / Accepted: 8 April 2018 / Published online: 23 April 2018

(c) The Author(s) 2018

\begin{abstract}
Purpose An integrated population pharmacokinetic (popPK) model was developed to describe the pharmacokinetics (PK) of tyrosine kinase inhibitor cabozantinib in healthy volunteers (HVs) and patients with various cancer types and to identify any differences in cabozantinib PK across these populations.

Methods Plasma concentration data used to develop the popPK model were obtained from nine clinical trials (8072 concentrations from $1534 \mathrm{HVs}$ or patients) of cabozantinib in HVs and patients with renal cell carcinoma (RCC), medullary thyroid carcinoma (MTC), glioblastoma multiforme, castration-resistant prostate cancer, or other advanced malignancies.

Results PK data across studies were adequately characterized by a two-compartment disposition model with dual first- and zero-order absorption processes and first-order elimination. Baseline demographic covariates (age, weight, gender, race, and cancer type) were generally predicted to have a small-to-moderate impact on apparent clearance (CL/F). However, MTC cancer type did show an approximately $93 \%$ higher $\mathrm{CL} / F$ relative to HVs following chronic dosing, resulting in approximately 40-50\% lower predicted steady-state cabozantinib plasma concentrations.

Conclusion This popPK analysis showed cabozantinib CL/ $F$ values to be higher for patients with MTC and may account for the higher dosage required in this patient population (140-mg) to achieve plasma exposures comparable to those in patients with RCC and other tumor types administered a 60-mg cabozantinib tablet dose. Possible factors that may underlie the higher cabozantinib clearance observed in MTC patients are discussed.
\end{abstract}

Keywords Cabozantinib $\cdot$ Population pharmacokinetics $\cdot$ Cancer types

\section{Introduction}

Cabozantinib is a tyrosine kinase inhibitor (TKI) targeting multiple receptor tyrosine kinases implicated in tumor angiogenesis, invasion, and metastasis, including MET (hepatocyte growth factor receptor), VEGFR2 (vascular endolethial growth factor receptor 2), AXL (GAS6 receptor), and RET (glial cell-derived neurotrophic factor receptor) [1]. The

Electronic supplementary material The online version of this article (https://doi.org/10.1007/s00280-018-3581-0) contains supplementary material, which is available to authorized users.

Steven Lacy

slacy@exelixis.com

1 Exelixis Inc., 210 East Grand Avenue, South San Francisco, CA 94080-0511, USA

2 Ann Arbor Pharmacometrics Group, Inc., Ann Arbor, MI, USA cabozantinib capsule formulation $\left(\right.$ Cometriq $\left.^{\circledR}\right)$ is approved at a dose of 140-mg-free base equivalents (FBE) daily in the USA for the treatment of progressive metastatic medullary thyroid cancer (MTC) and in the European Union (EU) for the treatment of progressive, unresectable locally advanced or metastatic MTC $[2,3]$. The cabozantinib tablet formulation $\left(\right.$ Cabometyx ${ }^{\mathrm{TM}}$ ) was subsequently approved at a dose of 60-mg FBE daily in the USA for the treatment of renal cell carcinoma (RCC) following anti-angiogenic therapy and in the EU following prior VEGF-targeted therapy $[4,5]$. Cabozantinib tablets are also being evaluated in a pivotal clinical study in patients with hepatocellular carcinoma at a $60-\mathrm{mg}$ FBE daily dose [6].

The cabozantinib tablet formulation (Cabometyx) and capsule formulation (Cometriq) were not bioequivalent following a single 140-mg dose in HVs [7]; the geometric least-squares mean (GLSM) for maximal plasma concentration $\left(C_{\max }\right)$ was $19 \%$ higher for the tablet formulation and the upper $90 \%$ confidence interval for the GLSM ratio for 
$C_{\max }(131.65 \%)$ slightly exceeded the $125 \%$ bioequivalence acceptance limit. However, the GLSM values for tablet and capsule formulations were similar $(<10 \%$ difference) for both area under the plasma concentration-time curve $\left(\mathrm{AUC}_{0-t}\right.$ and $\left.\mathrm{AUC}_{0-\infty}\right)$ measures, and the $90 \%$ CIs were $100-115 \%$ around the GLSM ratios.

The 140-mg FBE cabozantinib capsule dose used in the pivotal phase III study in patients with MTC was based on the maximally tolerated dose identified in a phase I study of cabozantinib in patients with MTC and other solid tumors [8]. In the pivotal phase III study, $79 \%$ of MTC patients (169 of 214) who received the 140-mg FBE cabozantinib capsule dose eventually dose-reduced [9]. Two protocoldefined cabozantinib dose reductions were allowed: from 140- to $100-\mathrm{mg} /$ day, and from 100- to 60-mg/day. Forty-two percent of MTC patients received $60-\mathrm{mg} /$ day as their final dose [10]. Exposure-response (ER) modeling suggested that the cabozantinib dose reductions from 140- to 100-mg and from 100- to 60-mg were not projected to result in a marked reduction in progression free survival (PFS) or in tumor lesion regrowth in patients with MTC $[11,12]$.

The 60-mg FBE cabozantinib tablet dose evaluated in the phase III study in patients with RCC was based on findings from a phase I study in patients with RCC of improved tolerance to study drug and evidence of clinical activity in patients who had dose-reduced from 140- to 60-mg [13]. Dose reductions to a 40 - or a 20 -mg daily dose were permitted in the pivotal phase III study in RCC patients to maintain treatment in response to drug-related adverse events (AEs). Although lower than the 140-mg capsule dose administered to MTC patients, the 60-mg cabozantinib tablet dose was associated with a high percentage of dose reductions in both the phase III study in RCC patients $(62 \% ; 206$ of 330) [14] and in a phase III study in patients with castrateresistant prostate cancer (CRPC) $(74 \%$; 505 of 682) [15]. ER modeling suggested cabozantinib exposures associated with a simulated 60-mg dose in RCC patients would result in slightly greater decreases in PFS, median percent change of tumor size from baseline, and best overall response rate (based on target lesion) relative to simulated 40- or 20-mg starting doses [16].

A popPK analysis was previously performed on pooled data for 289 cabozantinib-treated cancer patients (including MTC) receiving daily administration of the cabozantinib capsule formulation at a dose of 140-mg FBE/ day, except for five subjects that were dosed at 200-mg FBE/day [11]. The data were adequately described by a 1-compartment model with first-order absorption and first-order elimination with a small lag time. The mean $\mathrm{CL} / F$ and apparent volume of distribution of the central compartment $(\mathrm{Vc} / F)$ values estimated for a typical White male subject were 4.42 [standard error (SE)\%: $2.98 \%$ )] $\mathrm{L} / \mathrm{h}$ and 349 (SE\%: 2.73\%) L, respectively, resulting in an estimated effective half-life of $55 \mathrm{~h}$. The inter-subject variability (IIV) for CL/F (CV\%) was 35\%.

A popPK analysis of cabozantinib was subsequently performed using data collected from 282 patients with RCC and 63 normal HVs following oral administration of doses of 20-, 40-, and 60-mg [17]. A two-compartment disposition model with dual (fast and slow) lagged firstorder absorption processes adequately characterized the concentration-time profile of cabozantinib in HVs and patients with RCC. The mean CL/F and terminal-phase volume of distribution $\left(V_{\mathrm{z}}\right)$ predicted for a typical White male subject were $2.23 \mathrm{~L} / \mathrm{h}(90 \%$ CI $2.13,2.34)$ and $319 \mathrm{~L}$ (SE\%: $2.7 \%$ ), respectively, resulting in an estimated terminal plasma half-life of approximately $99 \mathrm{~h}$. The IIV for $\mathrm{CL} / F$ was $46 \%$. These popPK modeling analyses indicated that cabozantinib CL/ $F$ was approximately twofold lower in RCC patients than in MTC patients, which is consistent with the comparable observed steady-state exposures $\left(C_{\text {trough,ss }}\right)$ in RCC and CRPC patients administered a 60-mg tablet dose and in MTC patients administered a 140-mg capsule dose [16]. Based on these apparent differences in cabozantinib PK observed across cancer patients with different tumor types, an integrated popPK model was developed with the pooled PK data from different patient populations and HVs to evaluate the potential impact of patient population, formulations, and doses on the PK of cabozantinib.

\section{Methods}

\section{Study design and data}

The popPK analysis was conducted using data from nine clinical studies of cabozantinib including two phase I studies in HVs [7], a phase I study in cancer patients with advanced malignances [8], phase II studies in patients with GB [18] and CRPC [19, 20], and phase III studies in patients with RCC [14], MTC [9], or CRPC [15]. The results of most of these studies have been previously published; and a summary of the study designs, dosages, and PK sampling schemes is presented in Table 1. All studies were conducted following the ethical principles of the Declaration of Helsinki and Good Clinical Practice guidelines. Written informed consent was obtained from all patients and HVs.

\section{Bioanalytical methods}

Plasma cabozantinib concentrations were measured using a validated liquid chromatographic-tandem mass spectrometry 
Table 1 Summary of clinical studies included in the integrated population pharmacokinetic model of cabozantinib

\begin{tabular}{|c|c|c|c|c|}
\hline Study no. (Reference) & Design & Patient population & Cabozantinib dose & $\begin{array}{l}\text { Planned pharmacokinetic } \\
\text { sampling }\end{array}$ \\
\hline XL184-001 [8] & $\begin{array}{l}\text { Phase 1, nonrandomized, } \\
\text { open-label FIH study }\end{array}$ & Mixed malignancies & $140-$ or $200-\mathrm{mg}$ & $\begin{array}{l}\text { Days } 1 \text { and 19: pre-dose, } 0.5 \text {, } \\
\text { 1, 2, } 4,8 \text {, and } 24 \mathrm{~h} \\
\text { Day 5: pre-dose and } 4 \mathrm{~h} \\
\text { Days } 15 \text { and 29: pre-dose }\end{array}$ \\
\hline XL184-010 [7] & $\begin{array}{l}\text { Phase } 1 \text {, crossover BE } \\
\text { study of tablet and } \\
\text { capsule }\end{array}$ & Healthy volunteer & $140-\mathrm{mg}$ & $\begin{array}{l}\text { Pre-dose, } 0.5,1,2,3,4,5,6 \\
\text { 8, 10, 12, 14, 24, 48, 72, } \\
\text { 120, 168, 240, 288, 336, } \\
\text { 408, and } 504 \mathrm{~h}\end{array}$ \\
\hline XL184-020 [7] & Phase $1, \mathrm{PK}$ of tablet & Healthy volunteer & $20-, 40-, 60-\mathrm{mg}$ & $\begin{array}{l}\text { Pre-dose, } 0.5,1,2,3,4,5, \\
6,8,10,12,14,24,48,72, \\
\text { 120,168, 240, 288, 336, } \\
408, \text { and } 504 \mathrm{~h}\end{array}$ \\
\hline XL184-201 [18] & $\begin{array}{l}\text { Phase 2, multicenter, open } \\
\text { label }\end{array}$ & $\begin{array}{l}\text { Progressive glioblastoma } \\
\text { multiforme }\end{array}$ & 140-mg QD & $\begin{array}{l}\text { Each Cycle } 28 \text { days } \\
\text { Cycle } 1 \text { : pre-dose, and } 4 \mathrm{~h} \text { on } \\
\text { Days } 1 \text { and } 15 \\
\text { Cycle } 2 \text { : pre-dose and } 4 \mathrm{~h} \text { on } \\
\text { Days } 29 \text { and } 43 \\
\text { Cycle } 3 \text { and beyond: pre-dose } \\
\text { on day } 1\end{array}$ \\
\hline XL184-203 [19, 20] & $\begin{array}{l}\text { Phase } 2 \text {, randomized dis- } \\
\text { continuation study }\end{array}$ & $\begin{array}{l}\text { Castration-resistant prostate } \\
\text { cancer }\end{array}$ & $\begin{array}{l}\text { RDT: } 100-\mathrm{mg} \text { QD } \\
\text { NRE: 40- or 100-mg } \\
\text { QD }\end{array}$ & $\begin{array}{l}\text { RDT: pre-dose after "even" } \\
\text { weeks after week } 12 \text { lead- } \\
\text { in, or early termination or } \\
\text { adverse event } \\
\text { NRE; pre-dose week } 1 \text { day } 1 \text {; } \\
\text { pre-dose end of week } 3 \text { and } \\
6,12,18 \text {, and } 24 \text {, unsched- } \\
\text { uled, early termination or } \\
\text { adverse event }\end{array}$ \\
\hline XL184-301 [9] & $\begin{array}{l}\text { Phase 3, randomized, } \\
\text { double-blind, placebo- } \\
\text { controlled }\end{array}$ & $\begin{array}{l}\text { Metastatic medullary thy- } \\
\text { roid cancer }\end{array}$ & 140-mg QD & $\begin{array}{l}\text { Cycle } 1 \text {, day } 1 \text { : pre-dose and } \\
2,4 \text {, and } 6 \mathrm{~h} \\
\text { Cycle } 2 \text {, day } 29: \text { pre-dose and } \\
2,4 \text {, and } 6 \mathrm{~h}\end{array}$ \\
\hline $\begin{array}{l}\text { XL184-306 } \\
\text { [NCT01522443] }\end{array}$ & $\begin{array}{l}\text { Phase 3, randomized, } \\
\text { double-blind, controlled } \\
\text { versus prednisone }\end{array}$ & $\begin{array}{l}\text { Castration-resistant prostate } \\
\text { cancer }\end{array}$ & 60-mg QD & $\begin{array}{l}\text { Week } 1 \text { day } 1 \text {, Week } 4 \text { day } 1 \\
\text { Week } 7 \text { day } 1 \text {, Week } 13 \text { day } 1\end{array}$ \\
\hline XL184-307 [15] & $\begin{array}{l}\text { Phase 3, randomized, } \\
\text { double-blind, controlled } \\
\text { versus prednisone }\end{array}$ & $\begin{array}{l}\text { Castration-resistant prostate } \\
\text { cancer }\end{array}$ & 60-mg QD & $\begin{array}{l}\text { End of Week } 3 \text { and End of } \\
\text { Week } 12\end{array}$ \\
\hline XL184-308 [14] & $\begin{array}{l}\text { Phase } 3 \text {, randomized, con- } \\
\text { trolled versus everolimus }\end{array}$ & Renal cell carcinoma & 60-mg QD & $\begin{array}{l}\text { Days } 29 \text { and } 57 \text { approxi- } \\
\text { mately eight or more hours } \\
\text { after prior evening's dose }\end{array}$ \\
\hline
\end{tabular}

$B E$ bioequivalence, $F I H$ first-in-human, $Q D$ once daily, $R D T$ randomized discontinuation trial, $N R E$ nonrandomized expansion

method. The lower limit of quantitation (LLOQ) was $0.5 \mathrm{ng} /$ $\mathrm{mL}[21]$.

\section{Analysis of data files}

Source data in SAS format included information such as PK sample concentrations, PK sample dates and times, dose amounts with dates and times, and patient demographics and covariates. NONMEM ${ }^{\circledR}$ (Version 7) ready data sets were constructed using SAS (Version 9.3), S-plus (Version 8.2) or R (version 3.0.2).
Missing PK drug concentrations, if any, were documented and excluded from the analysis. Drug concentrations which were below the level of quantification (BLQ) were retained in the analysis data set but excluded from the analysis, because the number of BLQ samples was small $(<1 \%)$.

Baseline covariate values were assigned using covariate information collected prior to the first dose of study medication. Covariate values closest to the first dose of study medication were used first; however, if covariate information was not available immediately before study drug administration (e.g., pre-dose on day 1), then covariate information from 
Table 2 Baseline demographics and covariates in each clinical study used in the integrated population pharmacokinetic model of cabozantinib

\begin{tabular}{|c|c|c|c|c|c|c|c|c|c|c|}
\hline Study & 001 & 010 & 020 & 201 & 203 & 301 & 306 & 307 & 308 & Total \\
\hline$N$ of subjects & 40 & 77 & 63 & 39 & 284 & 210 & 41 & 498 & 282 & 1534 \\
\hline \multicolumn{11}{|l|}{ Sex } \\
\hline Male (\%) & $31(77.5)$ & 32 (41.6) & $33(52.4)$ & $26(66.7)$ & $284(100)$ & $146(69.5)$ & $41(100)$ & $498(100)$ & $222(78.7)$ & 1313 (85.6) \\
\hline Female (\%) & $9(22.5)$ & $45(58.4)$ & $30(47.6)$ & $13(33.3)$ & $0(0)$ & $64(30.5)$ & $0(0)$ & $0(0)$ & $60(21.3)$ & $221(14.4)$ \\
\hline \multicolumn{11}{|l|}{ Race } \\
\hline White (\%) & $35(87.5)$ & $74(96.1)$ & $62(98.4)$ & 33 (84.6) & $246(86.6)$ & $188(89.5)$ & 34 (82.9) & $380(76.3)$ & 231 (81.9) & 1283 (83.6) \\
\hline Black (\%) & $2(5)$ & $2(2.6)$ & $1(1.6)$ & $3(7.7)$ & $15(5.3)$ & $1(0.5)$ & $4(9.8)$ & $9(1.8)$ & $5(1.8)$ & $42(2.7)$ \\
\hline Asian (\%) & $1(2.5)$ & $0(0)$ & $0(0)$ & $1(2.6)$ & 14 (4.9) & $9(4.3)$ & $1(2.4)$ & $1(0.2)$ & $19(6.7)$ & $46(3.0)$ \\
\hline Other $(\%)$ & $2(5)$ & $1(1.3)$ & $0(0)$ & $1(2.6)$ & $9(3.2)$ & $5(2.4)$ & $2(4.9)$ & $3(0.6)$ & $16(5.7)$ & $39(2.5)$ \\
\hline NA $(\%)$ & $0(0)$ & $0(0)$ & $0(0)$ & $1(2.6)$ & $0(0)$ & $7(3.3)$ & $0(0)$ & $105(21.1)$ & $11(3.9)$ & $124(8.1)$ \\
\hline \multicolumn{11}{|l|}{ Population } \\
\hline Healthy (\%) & $0(0)$ & 77 (100) & $63(100)$ & $0(0)$ & $0(0)$ & $0(0)$ & $0(0)$ & $0(0)$ & $0(0)$ & $140(9.1)$ \\
\hline CRPC (\%) & $0(0)$ & $0(0)$ & $0(0)$ & $0(0)$ & $284(100)$ & $0(0)$ & $41(100)$ & $498(100)$ & $0(0)$ & $823(53.7)$ \\
\hline $\mathrm{RCC}(\%)$ & $0(0)$ & $0(0)$ & $0(0)$ & $0(0)$ & $0(0)$ & $0(0)$ & $0(0)$ & $0(0)$ & $282(100)$ & $282(18.4)$ \\
\hline $\operatorname{MTC}(\%)$ & $0(0)$ & $0(0)$ & $0(0)$ & $0(0)$ & $0(0)$ & $210(100)$ & $0(0)$ & $0(0)$ & $0(0)$ & $210(13.7)$ \\
\hline GB $(\%)$ & $0(0)$ & $0(0)$ & $0(0)$ & 39 (100) & $0(0)$ & $0(0)$ & $0(0)$ & $0(0)$ & $0(0)$ & $39(2.5)$ \\
\hline Other $^{\mathrm{a}}(\%)$ & $40(100)$ & $0(0)$ & $0(0)$ & $0(0)$ & $0(0)$ & $0(0)$ & $0(0)$ & $0(0)$ & $0(0)$ & $40(2.6)$ \\
\hline \multicolumn{11}{|l|}{ Formulation } \\
\hline Capsule (\%) & $40(100)$ & $75^{\mathrm{b}}$ & $0(0)$ & $39(100)$ & $284(100)$ & $210(100)$ & $0(0)$ & $0(0)$ & $0(0)$ & $648(42.2)$ \\
\hline Tablet (\%) & $0(0)$ & & $63(100)$ & $0(0)$ & $0(0)$ & $0(0)$ & $41(100)$ & $498(100)$ & $282(100)$ & $959(62.5)$ \\
\hline \multicolumn{11}{|l|}{ Body weight } \\
\hline Range (kg) & $53.4-116$ & $46.1-106$ & $58.1-113.5$ & $52-125.3$ & $50.3-182.9$ & $30.4-137.9$ & $57.5-190.7$ & $49.7-140^{\mathrm{c}}$ & $48.1-155.7$ & $30.4-190.7$ \\
\hline Mean (SD) & 82.8 (15.9) & $71.9(11.5)$ & $76.4(11.8)$ & $81.4(18.3)$ & $90.2(18.6)$ & $72.9(18)$ & $89.3(23.1)$ & $83.3(14.0)$ & $81.9(17.0)$ & $82.1(17.3)$ \\
\hline Mean & 83.0 & 71.9 & 76.5 & 79.4 & 87.8 & 71.3 & 84.8 & 82.3 & 80.4 & 81.0 \\
\hline \multicolumn{11}{|l|}{ Age } \\
\hline Range (yrs) & $23-71$ & $18-55$ & $19-54$ & $20-67$ & $43-87$ & $20-86$ & $48-79$ & $35-87$ & $32-86$ & $18-87$ \\
\hline Mean (SD) & $56.0(11.0)$ & $39.3(9.7)$ & 36.9 (8.6) & $48.6(13.5)$ & $66.3(8.8)$ & $54.7(13.3)$ & $64.8(6.4)$ & 68.7 (7.6) & $61.6(9.5)$ & $61.3(13.1)$ \\
\hline Median & 57 & 39 & 38 & 53 & 67 & 55 & 65 & 69 & 62 & 64 \\
\hline
\end{tabular}

$C R P C$ castrate resistant prostate cancer, $G B$ glioblastoma multiforme, $M T C$ medullary thyroid cancer, $N$ number, $N A$ not available, $R C C$ renal cell carcinoma, $S D$ standard deviation

${ }^{a}$ Unknown mixed cancer type in Study 001

${ }^{\mathrm{b}}$ Study 010 is a cross-over study of capsule versus tablet formulations. The total percentage of subjects on tablet and capsule do not add up to $100 \%$ due to due to the crossover design in which each subject received both formulations.

${ }^{\mathrm{c}}$ Six subjects in study 307 had missing weight information

a previous visit (e.g., screening) was used. A summary of the demographic characteristics and relevant covariates for HVs and cancer patients included in the integrated popPK analysis is found in Table 2.

\section{Population PK model}

Analyses were performed using the nonlinear mixed effect modeling as implemented in NONMEM (version 7.3 ICON Development Solutions, Ellicott City, MD, USA). Estimation methods used included first-order conditional estimation with interaction (FOCEI), iterative two-stage (ITS), stochastic approximation expectation maximization (SAEM), and importance sampling method (IMP).

\section{Base model}

The PK Base Model was developed initially using only the exposure data from the HV and RCC studies, and then subsequently with the full integrated data set. Structural models evaluated were one- and two-compartment disposition models with first-order elimination, first-order absorption, and absorption lag time. The previous reports indicated that the concentration-time profile showed multiple peaks suggesting enterohepatic recirculation or multiple absorption sites [7, 21]; therefore, other models were considered such as dual lagged first-order absorption models or transit compartment models with increasing number of transit compartments. 
Inter-individual variability (IIV) of the PK parameters was incorporated using a log normal random effects model:

$\theta_{\mathrm{i}}=\theta_{\mathrm{T}} \cdot e^{\left(\eta_{\mathrm{i}}\right)}$,

where $\theta_{\mathrm{i}}$ is the individual value of the PK parameter (e.g., $\mathrm{CL} / F), \theta_{\mathrm{T}}$ is the typical value of the parameter, and $\eta_{\mathrm{i}}$ is the inter-individual random effect assumed to have a normal distribution with a mean of zero and variance of $\omega^{2}$. The vector of IIV random effects had a variance-covariance matrix $\Omega$. A full-block $\Omega$ was estimated. Reductions to fullblock covariance structure were considered if instability in the model was encountered.

Residual variability (RV), a composite measure of assay error, dose/sample time collection errors, model misspecification, and any other unexplained variability within a subject, was initially modeled using the log-transformed additive error model:

$\ln \left(Y_{i j}\right)=\ln \left(C_{i j}\right)+\varepsilon_{i j}$,

where $Y_{i j}$ denotes the observed drug concentration for the $i$ th individual at time $t_{j}, C_{i j}$ denotes the corresponding predicted concentration based on the PK model, and $\varepsilon_{i j}$ denotes the residual random variable, which is assumed to have a normal distribution with a zero mean and variance $\sigma^{2}$. Other residual error models were explored if patterns were observed in the individual weighted residual (IWRES) versus individual predicted value (IPRED) plot.

\section{Covariate model}

The covariate analysis was performed using a full model approach [22, 23]. Covariates were pre-specified based upon clinical judgment and mechanistic plausibility and included: age, weight, sex, race, and population (cancer patient type including RCC, CRPC, MTC, GB, and advanced malignancy or $\mathrm{HV}$ ) on $\mathrm{CL} / F$ and $\mathrm{Vc} / F$. The full model was constructed by including simultaneously all pre-specified covariates of interest into the base model.

The relationship between continuous covariates and the typical value of PK parameters was described using power models:

$\theta_{\mathrm{TV}, i j}=\theta_{\mathrm{REF}}\left(\frac{x_{i j}}{x_{\mathrm{REF}}}\right)^{\theta_{x}}$

where $\theta_{\mathrm{REF}}$ and $\theta_{x}$ are the fixed-effect parameters and $x_{\mathrm{REF}}$ is a reference value of the covariate $\chi_{i j}$. The approximate median value was used for $\chi_{\mathrm{REF}}$. The relationship between categorical covariate and typical value of $\mathrm{PK}$ parameters was modeled as follows:

$\theta_{\mathrm{TV}, i j}=\theta_{\mathrm{REF}} \cdot \exp \left(\theta_{x} \cdot x_{i j}\right)$, where $\theta_{\mathrm{REF}}$ and $\theta_{x}$ are fixed-effect parameters and $\chi_{i j}$ is the indicator variable with values of 1 or 0 . To prevent negative parameter values in simulations, $\theta_{x}$ is the $\log$ of the fractional change in the typical value for a categorical covariate.

Plots of the individual random effect values versus covariate values were generated to evaluate if inclusion of the covariate effects reduced or eliminated trends in the random effects/PK parameters. In addition, box plots of the $\eta$ values versus dose and study were generated to evaluate adequacy of pooling studies for this analysis.

At each key step in the model development, a complete battery of diagnostic plots was generated. Standard goodness-of-fit plots were used to assess lack-of-fit. Different structural models were considered if the initial model did not adequately describe the integrated cabozantinib concentration-time data.

\section{Posterior predictive check}

An internal posterior predictive check (PPC) was performed to assess the predictive performance of the popPK models [24]. A smoothed parametric bootstrap procedure was implemented to account for uncertainty in the parameter estimates. A total of 500 sets of population parameter values were generated using the multivariate normal distribution with the mean vector set to the population parameter estimates and the covariance matrix set to that of the final model. These values were used to simulate a data set replicating study design, sample size, and covariate distributions from the observed data set. The PPC statistics including the median, and 10th and 90th percentiles were computed at nominal time points for both the observed and each simulated data set. Prediction intervals were constructed based on the 5th and 95th percentiles of the simulated distributions of the PPC statistics.

\section{Results}

\section{Data}

The final data set contained a total of 8072 plasma concentrations from 1534 patients and HVs. All BLQ samples were excluded from the analysis, since the percentage of samples that were BLQ was small $(<1 \%)$.

The majority of subjects were male (85.6\%), as four of the nine studies enrolled patients with CRPC, and white $(83.6 \%)$. Body weights and ages were generally consistent across the studies, except for the HV studies which had younger subjects due to the exclusion criteria. The singledose clinical pharmacology studies in HVs and the phase 
I safety study that enrolled patients with advanced mixed malignancies (XL184-001) contained intensive sampling which allowed for full characterization of the cabozantinib PK profile. The phase II and III clinical studies in cancer patients administered cabozantinib daily provided sparse PK sampling data.

\section{Population pharmacokinetic modeling}

Supplemental Table 1 lists the key steps in development of the popPK model. Initially, the base popPK model was developed utilizing only exposure data from studies enrolling HVs or RCC patients. A two-compartment disposition model and a dose-dependent dual absorption model containing two lagged first-order absorption processes (dosedependent fast and slow) adequately characterized the cabozantinib PK data. However, numerical problems and long run times were encountered when the model was fit to the fully integrated data set that contained all study populations and covariates [including demographics (age, weight, sex, and race) and cancer population effects (RCC, CRPC, MTC, GB, and advanced malignancies)] (full model). Therefore, the dual first-order absorption model was replaced by a model with dual first-order and zero-order absorption [full modified (FM) model]. The first-order absorption process including a lag time and a dose-dependent effect on the absorption rate constant (Ka) was described using a power model. In addition, capsule formulation was included as a structural covariate on Ka and overall bioavailability based on prior knowledge from the capsule-tablet bioequivalence study (XL184-020; [7]).

The FM model was stable and adequately described the PK data in the fully integrated cabozantinib PK data set across different studies (Supplemental Fig. 1). Close inspection of the model output suggested that the magnitude of demographic and cancer population-specific covariate effects on cabozantinib PK was small, except for MTC patient population who had a substantially higher estimated $\mathrm{CL} / F$. To determine the significance of covariate effects, and the MTC covariate effect on $\mathrm{CL} / F$, two additional ad hoc model runs were performed relative to the FM model: (1) all covariates except for dose on k12 and capsule on Ka and F1 were removed (BASE) and (2) cancer-type covariates on $\mathrm{CL} / F$ and $\mathrm{Vc} / F$ were removed (FMECT). The OFV was increased 401.2 units when all covariates (i.e., demographic and cancer type) were removed (comparing BASE to FM), suggesting significant effects of demographic covariates and cancer types together. The OFV was 305.9 units higher when cancer-type covariates were excluded (comparing FMECT to FM), indicating a significant effect of one or more cancer types on the PK of cabozantinib. Furthermore, the OFV for BASE model was 95.4 units higher than the OFV for the FMECT model, suggesting that some other demographic covariates were also significant, but their effect was less than cancer-type covariates. Goodness-of-fit plots showed all three models provided reasonable fit to the data, but there was some lack-of-fit between observed and predicted geometric means concentrations for MTC patients for the BASE and FMECT models, with MTC patients having a higher estimated CL/F (Fig. 1). Only after including cancer type as a covariate on $\mathrm{CL} / F$ (FM model) did the trend between IIR on CL/F and cancer patient population resolve (Fig. 2). Thus, the FM model was determined to be the final model.

Cabozantinib PK parameter estimates for all three models are shown in Table 3. For the FM model, the transformed estimates $(90 \% \mathrm{CI})$ for $\mathrm{CL} / F$ and $\mathrm{Vc} / F$ were 2.478 (2.257, 2.721) L/h and 187 (156.3, 223.9) L, respectively. Demographic covariates (age, weight, sex, and race) generally showed minimal effect on $\mathrm{CL} / F$ and $\mathrm{Vc} / F$, although race covariate Black did result in an approximately $30 \%$ increase in CL/F. Cancer-type covariates RCC, CRPC, GB and Other showed minimal effects on $\mathrm{CL} / F$ and $\mathrm{Vc} / F$, whereas patients with MTC cancer type were predicted to have approximately 93\% higher CL/F relative to HVs. Thus, when compared to HVs at the same dosage, patients with MTC would have approximately 40 and $50 \%$ lower steady-state maximal $\left(C_{\text {max,ss }}\right)$ and minimal $\left(C_{\text {min,ss }}\right)$ exposures, respectively (Fig. 3).

The predictive performance of the three models for patients with MTC stratified by day of study demonstrated that the lack-of-fit was most apparent on day 29 of the study for the BASE and FMECT models (Supplemental Fig. 2). These findings suggested that the day 29 concentration data and reduced accumulation relative to that expected from $\mathrm{HVs}$ are largely responsible for driving the increase in CL/F for patients with MTC. To confirm this finding, an additional ad hoc run was performed using the FM model to a re-fit data set including only day 1 data. In this model, the population effect for MTC was - 0.312 (90\% CI - 0.824, 0.201 ), suggesting that CL/F on day 1 for MTC patients was not significantly different from HVs. Model prediction using this ad hoc model run remained reasonable for PK data on day 1 , but, when the predictions from the ad hoc model were applied to day 29, the simulated data were much higher than observed PK data for MTC patients (Supplemental Fig. 3). Using the full data set and the FM model which included a covariate for MTC patients, the fit was substantially improved on day 29 , while an acceptable fit was maintained on day 1 (Supplemental Fig. 2).

\section{Discussion}

Cabozantinib is a TKI approved for the treatment of MTC and RCC [2-5]. Although the formulations and dosages are different for MTC (140-mg/day Cometriq) and RCC (60-mg/ 


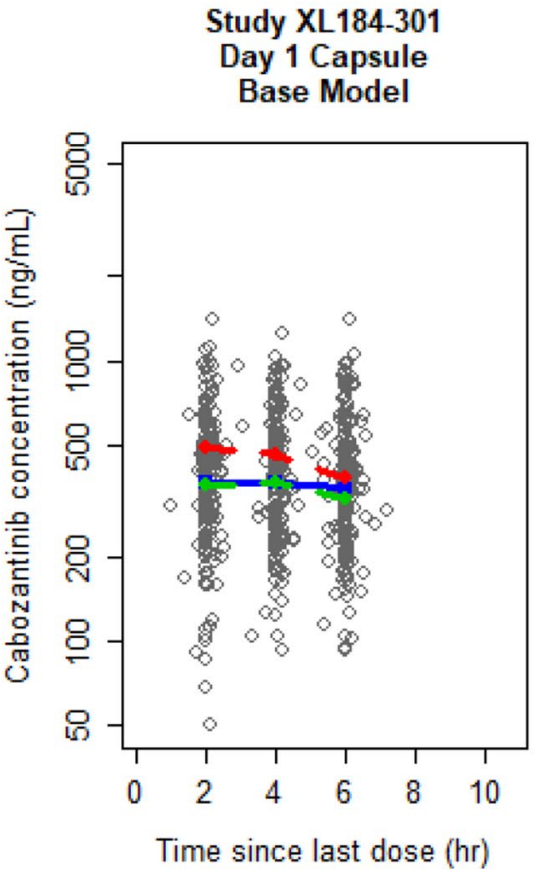

Study XL184-301

Day 29 Capsule

Base Model

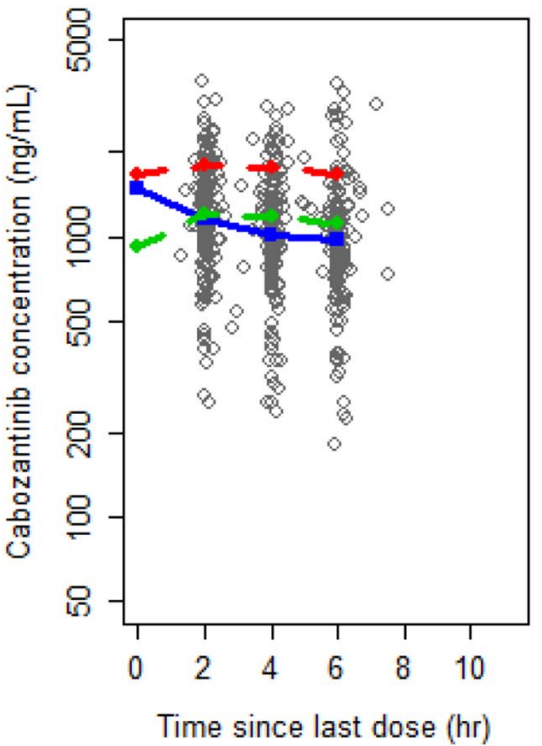

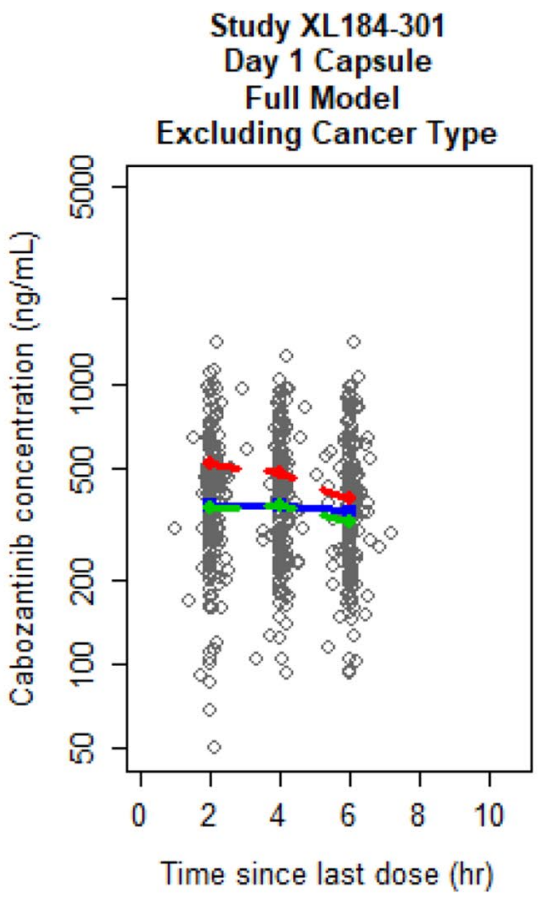

Study XL184-301

Day 29 Capsule

Full Model

Excluding Cancer Type

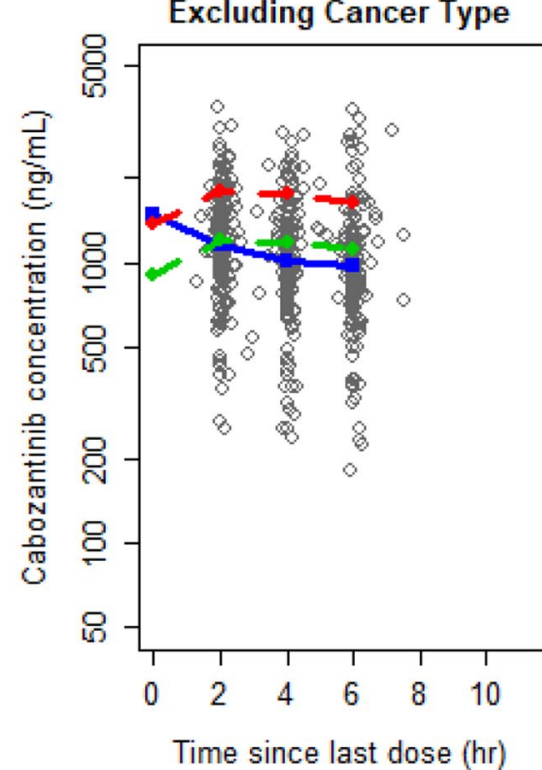

Study XL184-301

Day 1 Capsule

Full Model

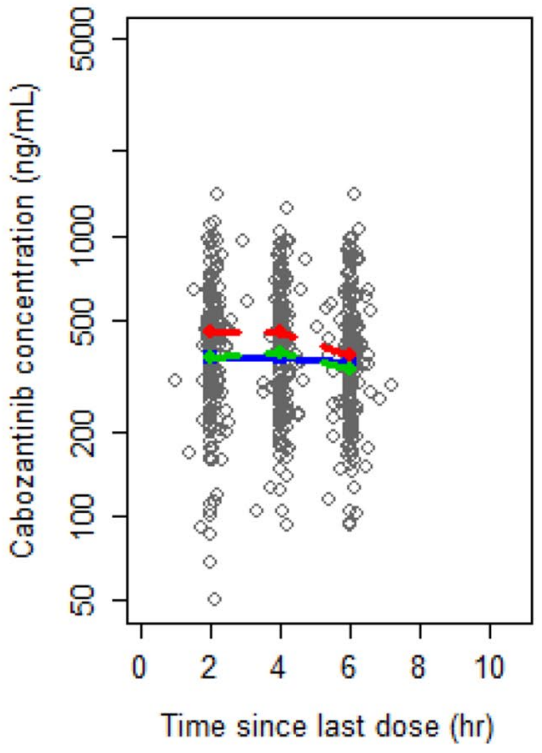

Study XL184-301

Day 29 Capsule Full Model

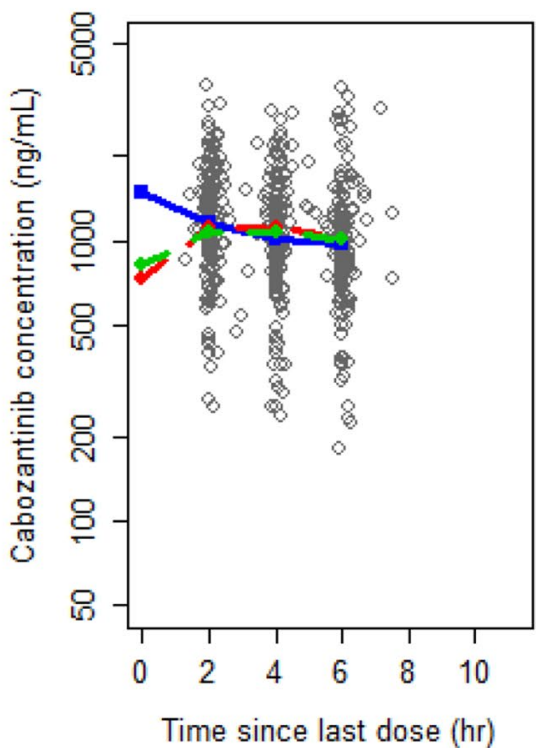

Fig. 1 Comparison of goodness-of-fit plots for patients with medullary thyroid cancer on day 1 and day 29 of study XL184-301. Solid blue, red-dashed, and green-dashed lines correspond to geometric

day Cabometyx) and dose adjustments and interruptions were allowed in the respective phase III studies, the resultant cabozantinib steady-state exposures in the pivotal phase III studies were comparable for the two patient populations [16]. Findings from popPK analyses subsequently showed mean observed, typical individual predicted (PREDs), and individually predicted (IPREDs) concentrations, respectively

that cabozantinib $\mathrm{CL} / F(\mathrm{CV} \%)$ in MTC patients $[4.4 \mathrm{~L} / \mathrm{h}$ (35\%)] was twofold higher than in RCC patients [2.2 L/h $(46 \%)][11,17]$, suggesting an apparent difference in cabozantinib clearance in patients with different tumor types. 

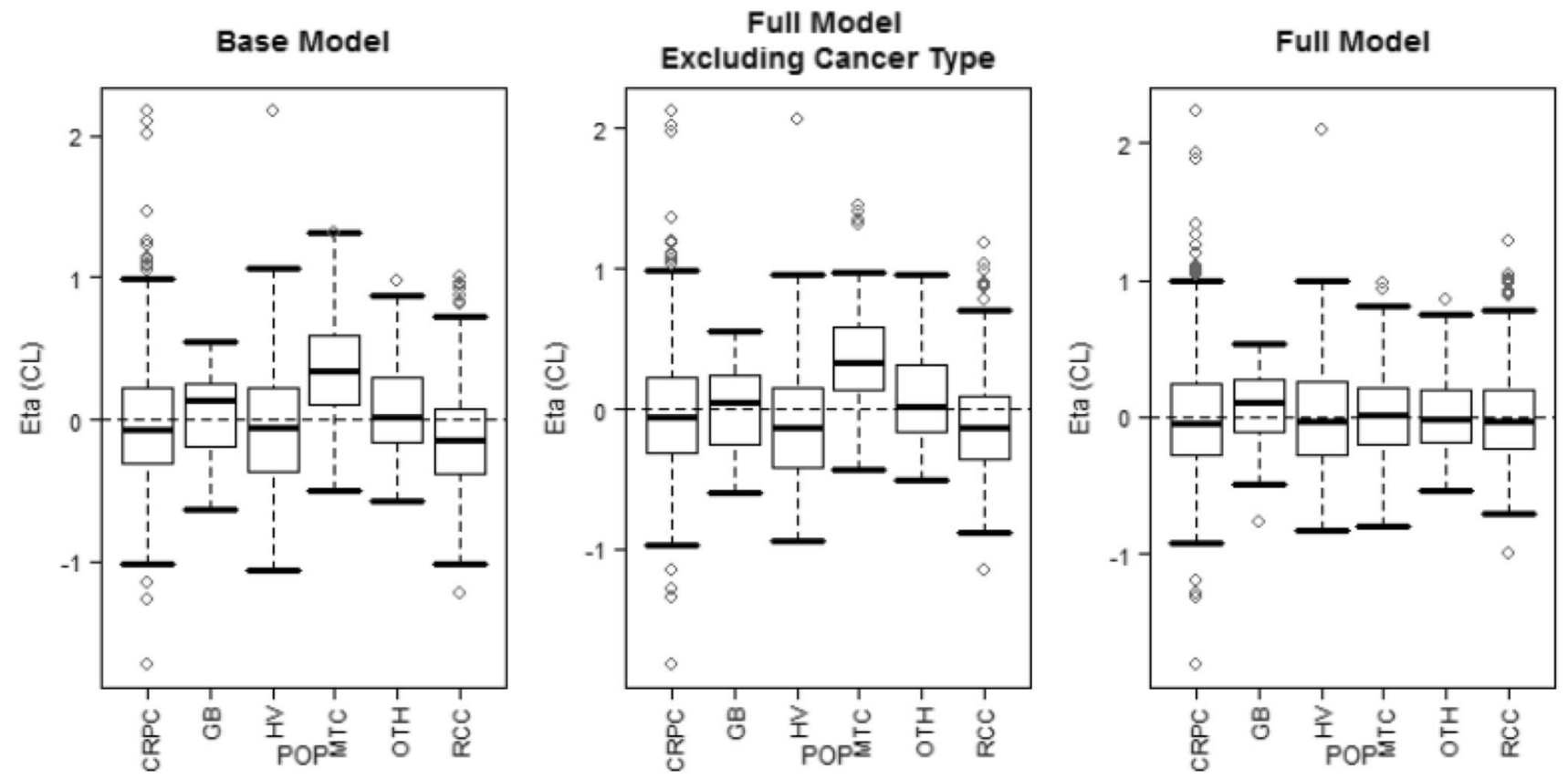

Fig. 2 Inter-individual random effect (Eta) on CL/ $F$ versus subject population. The boxes represent median and 25 th and 75 th percentiles. The bars represent 5th and 95th percentiles The open circles represent individual values outside the 5 th and 9 th percentiles. $C L$

To examine the extent to which demographic covariates could explain heterogeneity in the PK parameters across cancer populations, an integrated population PK analysis of cabozantinib was conducted using exposure data from HVs and cancer patients with different types of malignancies (ie, MTC, RCC, CRPC, GB). This analysis included data from nine clinical studies (three phase I, two phase II and four phase III) for a total of 8072 cabozantinib concentration records from 1534 subjects. A two-compartment model with first-order elimination and a dual absorption (first-order + zero-order) process adequately described the observed cabozantinib PK data.

The FM model which incorporated demographic covariates (age, body weight, sex, and race) and type of cancer malignancy (RCC, CRPC, MTC, GB, and other malignancies) on cabozantinib CL/F and $\mathrm{Vc} / F$ was evaluated. While most covariate effects (including patient demographics) included in the FM model had small-to-moderate effects on cabozantinib PK parameters and exposure metrics, MTC cancer-type led to a $>90 \%$ increase in CL/F. Ad hoc analyses showed that the cabozantinib concentrations at day 29 were primarily driving the increase in CL/F in MTC patients in pivotal phase III study XL184-301. MTC patients had lower steady-state plasma concentrations at day 29 than anticipated for the given dose relative to patients with other cancer types or HVs and suggested that lower observed accumulation could be due to higher clearance in this patient clearance, $C R P C$ castrate-resistant prostate cancer, $G B$ glioblastoma multiforme, $H V$ healthy volunteer, $M T C$ medullary thyroid cancer, OTH other cancer types in Study XL184-001, POP population, RCC renal cell carcinoma

population. Possible reasons for the large increase in cabozantinib clearance at steady state for MTC patients evident in the integrated popPK analysis were explored, including differences in treatment-emergent AEs, concomitant medications, and administered cabozantinib dose.

Diarrhea is a common treatment-related AE in cancer patients receiving cabozantinib [8, 9, 13-15], and the 140$\mathrm{mg}$ dose administered to MTC patients may be anticipated to result in a higher incidence and/or severity of diarrhea than a 60-mg dose given to RCC and CRPC subjects. As cabozantinib is considered to undergo enterohepatic recirculation [21], a decrease in the absorption fraction of cabozantinib typically reabsorbed via enterohepatic reabsorption due to treatment-related diarrhea may result in an apparent increase in cabozantinib clearance. The severity of diarrhea and possible effect on clearance would be anticipated to be greater in MTC patients administered a higher cabozantinib dose $(140-\mathrm{mg})$ than that given to patients with other tumor types $(60-\mathrm{mg})$. However, there was no marked difference in Grade 3/4 diarrhea in the subjects enrolled in the cabozantinib arm of the pivotal MTC study administered a 140-mg dose [16\% (34 of 214); [9]] and in the cabozantinib arm of the pivotal RCC study administered a $60-\mathrm{mg}$ dose [13\% (43 of 311); [14]].

In a separate popPK analysis [26], MTC patients were reported to have higher (67\% greater) oral clearance for another TKI (motesanib) relative to patients with 
Table 3 Parameter estimates for the final integrated population pharmacokinetic model of cabozantinib in patients with different cancer types

\begin{tabular}{|c|c|c|c|}
\hline \multirow[t]{2}{*}{ Parameter } & Base model (BASE) & $\begin{array}{l}\text { Full model excluding cancer } \\
\text { type (FMECT) }\end{array}$ & Full model (FM) \\
\hline & Transformed Estimate (90\% CI) & Transformed estimate (90\% CI) & Transformed estimate (90\% CI) \\
\hline $\mathrm{Ka}\left(\mathrm{h}^{-1}\right)$ & $0.804(0.576,1.123)$ & $0.846(0.606,1.182)$ & $0.979(0.679,1.411)$ \\
\hline $\begin{array}{l}\text { Duration of absorption for the zero-order } \\
\text { absorption process }(h)\end{array}$ & $2.435(1.966,3.016)$ & $2.441(92.096,2.843)$ & $2.4(2.01,2.866)$ \\
\hline $\mathrm{Cl} / F(\mathrm{~L} / \mathrm{h})$ & $2.457(2.396,2.519)$ & $2.553(2.482,2.625)$ & $2.478(2.257,2.721)$ \\
\hline $\mathrm{Vc} / F(\mathrm{~L})$ & $157.178(142.879,172.95)$ & $146.713(131.894,163.204)$ & $187.0(156.3 .223 .9)$ \\
\hline $\mathrm{Q} / F(\mathrm{~L} / \mathrm{h})$ & $30.154(27.743,32.786)$ & $30.118(27.883,32.525)$ & $31.213(28.732,33.92)$ \\
\hline $\mathrm{Vp} / F(\mathrm{~L})$ & $188.666(176.091,202.148)$ & $193.605(182.546,205.203)$ & $195.1(183.3,207.9)$ \\
\hline ALAG1 (h) & $0.789(0.763,0.815)$ & $0.777(0.752,0.804)$ & $0.784(0.757,0.812)$ \\
\hline $\begin{array}{l}\text { Fraction of dose in the first absorption depot } \\
F 1^{\mathrm{a}}\end{array}$ & $0.847(0.805,0.881)$ & $0.840(0.803,0.8720)$ & $0.854(0.819,0.884)$ \\
\hline Dose-dependent $\mathrm{Ka}^{\mathrm{c}}$ & $0.566(0.199-0.934)$ & $0.585(0.201,0.969)$ & $0.677(0.268,1.085)$ \\
\hline \multicolumn{4}{|l|}{ Covariates } \\
\hline Capsule on $\mathrm{Ka}^{\mathrm{b}}$ & $-0.211(-0.541,0.354)$ & $-0.300(-0.599,0.224)$ & $-0.579(-0.783,-0.183)$ \\
\hline $\begin{array}{l}\text { Capsule on overall relative oral } \\
\text { availability }^{\mathrm{b}}\end{array}$ & $-0.189(-0.205,-0.173)$ & $-0.183(-0.199,-0.167)$ & $-0.144(-0.162,-0.126)$ \\
\hline Age on $\mathrm{CL} / F^{\mathrm{c}}$ & & $-0.273(-0.367,-0.178)$ & $-0.162(-0.281,-0.042)$ \\
\hline Female on $\mathrm{CL} / F^{\mathrm{b}}$ & - & $-0.233(-0.29,-0.172)$ & $-0.230(-0.286,-0.17)$ \\
\hline Race (Black) on CL/F $F^{\mathrm{b}}$ & - & $0.249(0.085,0.439)$ & $0.301(0.139,0.486)$ \\
\hline Race (Asian) on $\mathrm{CL} / F^{b}$ & - & $-0.118(-0.233,0.013)$ & $-0.078(-0.192,0.052)$ \\
\hline Race (Other) on CL/F $F^{\mathrm{b}}$ & - & $-0.029(-0.161,0.124)$ & $-0.007(-0.0136,0.414)$ \\
\hline Weight on CL/F $F^{\mathrm{c}}$ & - & $-0.248(-0.373,-0.122)$ & $-0.028(-0.148,0.092)$ \\
\hline $\mathrm{RCC}$ on $\mathrm{CL} / F^{\mathrm{b}}$ & - & - & $-0.129(-0.217,-0.033)$ \\
\hline $\mathrm{CRPC}$ on $\mathrm{CL} / F^{\mathrm{b}}$ & - & - & $-0.009(-0.11,0.103)$ \\
\hline MTC on $\mathrm{CL} / F^{\mathrm{b}}$ & - & - & $0.928(0.738,1.136)$ \\
\hline $\mathrm{GB}$ on $\mathrm{CL} / F^{\mathrm{b}}$ & - & - & $0.216(0.02,0.449)$ \\
\hline Other malignancies on $\mathrm{CL} / F^{\mathrm{b}}$ & - & - & $0.178(0.003,0.384)$ \\
\hline Age on $\mathrm{Vc} / F^{\mathrm{c}}$ & & $-0.277(-0.459,-0.095)$ & $-0.012(-0.247,0.223)$ \\
\hline Female on $\mathrm{Vc} / F^{\mathrm{b}}$ & - & $0.165(0.023,0.327)$ & $0.11(-0.033,0.276)$ \\
\hline Race (Black) on $\mathrm{Vc} / F^{\mathrm{b}}$ & - & $-0.065(-0.362,0.372)$ & $-0.022(-0.334,0.438)$ \\
\hline $\operatorname{Race}($ Asian$)$ on $\mathrm{Vc} / F^{\mathrm{b}}$ & - & $0.125(-0.197,0.576)$ & $0.05(-0.278,0.528)$ \\
\hline Race (Other) on $\mathrm{Vc} / F^{\mathrm{b}}$ & - & $-0.018(-0.321,0.422)$ & $-0.059(-0.382,0.435)$ \\
\hline Weight on $\mathrm{Vc} / F^{\mathrm{c}}$ & - & $0.798(0.513,1.083)$ & $1.019(0.72,1.318)$ \\
\hline $\mathrm{RCC}$ on $\mathrm{Vc} / F^{\mathrm{b}}$ & - & - & $-0.63(-0.853,-0.069)$ \\
\hline CRPC on $\mathrm{Vc} / F^{\mathrm{b}}$ & - & - & $-0.241(-0.395,-0.049)$ \\
\hline MTC on $\mathrm{Vc} / F^{b}$ & - & - & $-0.07(-0.232,0.125)$ \\
\hline $\mathrm{GB}$ on $\mathrm{Vc} / F^{\mathrm{b}}$ & - & - & $-0.569(-0.72,-0.337)$ \\
\hline Other malignancies on $\mathrm{Vc} / F^{\mathrm{b}}$ & - & - & $-0.186(-0.372,0.055)$ \\
\hline Variance $^{\mathrm{d}}$ & - & - & - \\
\hline
\end{tabular}

$A L A G$, absorption lag time, $C I$ confidence interval, $C L / F$ apparent clearance, $C R P C$ castrate-resistant prostate cancer, $F 1$ fraction of dose split to the first absorption depot in a dual absorption model, $G B$ Glioblastoma multiforme, $K a$ absorption rate constant, $M T C$ medullary thyroid cancer, $Q / F$ apparent flow parameter between compartments, $R C C$ renal cell carcinoma $V c / F$ apparent volume of distribution of the central compartment, $V p / F$ apparent volume of distribution of the peripheral compartment

${ }^{a}$ Anti-logit transformation was used to obtain F1

${ }^{b}$ For categorical covariates (e.g., capsule), transformed estimates correspond to fractional change from the reference level

${ }^{c}$ untransformed values

${ }^{\mathrm{d}}$ Untransformed full model variance estimates $(90 \% \mathrm{CI}) \sigma 2=0.118(0.114,0.122) ; \omega^{2} \_\mathrm{Ka}=2.063(1.579,2.548) ; \omega^{2} \_\mathrm{CL} / F=0.202(0.185$, $0.218) ; \omega^{2}{ }_{-} \mathrm{Vc} / F=0.233(0.193,0.273) ; \omega^{2}{ }_{-} \mathrm{F} 1=0.466(0.385,0.546) ; \omega^{2}{ }_{-} \mathrm{CL} / F: \mathrm{Vc} / F=2.475(1.923,3.028)$, where $\omega^{2}$ is the variance-covariance matrix $(\Omega)$ of the inter-individual random effects $(\eta)$ in the pharmacokinetic parameter, and $\sigma$ the variance-covariance matrix of the intraindividual random effects $(\varepsilon)$ in the measurements 
Fig. 3 Impact of covariates on steady-state cabozantinib $\mathrm{CL} / F, C_{\min }$ and $C_{\max }$ relative to a reference White, male, $80 \mathrm{~kg}$, 60 year-old healthy subject. $\mathrm{CL} / F$, apparent clearance, Cmax,ss maximum plasma concentration at steady state, Cmin,ss minimum plasma concentration at steady state, $C R P C$ castrate-resistant prostate cancer, $G B$ glioblastoma multiforme, HAGE a 79-year-old subject, $H W T$ a subject with body weight of $112 \mathrm{~kg}, L A G E$ a 36 -year-old subject, $L W T$ a subject with body weight of $56 \mathrm{~kg}, M T C$ medullary thyroid cancer, $R C C$ renal cell carcinoma

differentiated thyroid cancer (DTC), in conjunction with a higher baseline incidence rate of diarrhea (68 and 6\% in MTC and DTC cohorts, respectively). Similar to cabozantinib, patients' disease type best accounted for inter-patient variability in motesanib CL/ $F$ of all covariates tested. However, incorporating diarrhea into the popPK model did not result in a significant improvement in the model fit, after accounting for the patients' disease type, and there was no difference in motesanib CL/ $F$ among MTC patients with severe, moderate, and mild diarrhea. In addition, both the motesantib and cabozantinib popPK analyses showed a minimal effect on $\mathrm{Vc} / F$ in MTC patients, whereas a reduction in oral bioavailability due to diarrhea would be expected to result in increases in both $\mathrm{CL} / F$ and $\mathrm{Vc} / F$. The mechanistic basis for the difference in motesanib CL/F between DTC and MTC patients was not identified.

Increased cabozantinib clearance in MTC patients at steady state could be related to treatment-emergent hypocalcemia, particularly in advanced MTC patients who undergo thyroidectomy when the parathyroid glands are also partially or completely removed resulting in decreased plasma parathyroid hormone levels. Hypocalcemia may affect drug clearance indirectly via stimulation of active vitamin $\mathrm{D}$ metabolite 1,25 dihydroxyvitamin $\mathrm{D}_{3}\left(1,25(\mathrm{OH})_{2} \mathrm{D}_{3}\right)$ synthesis, and subsequent induction of CYP3A4 by $1 \alpha, 25(\mathrm{OH})_{2} \mathrm{D}_{3}[27,28]$. Since cabozantinib is metabolized by CYP3A4 [29], hypocalcemia was considered as a potential contributing factor in reducing cabozantinib clearance in MTC patients. Although clinical laboratory-defined hypocalcemia was identified in $52 \%$ of MTC patients receiving cabozantinib in the pivotal phase III study XL184-301 [2], and in fewer MTC patients receiving placebo in the same study (27\%), overall no evidence of altered calcium levels was noted in patients with MTC compared to other cancers to suggest that hypocalcemia was responsible for increased cabozantinib clearance in this population.

Differences in concomitant medication use, particularly administration of strong CYP3A4 inducers in MTC patients, could have resulted in the increased cabozantinib clearance observed in the MTC patient population. However, only $1.4 \%$ of patients ( 3 of 207 total) were reported to have used a concomitant strong CYP3A4 inducer in the MTC phase III study of cabozantinib [11]. Cabozantinib is also a substrate
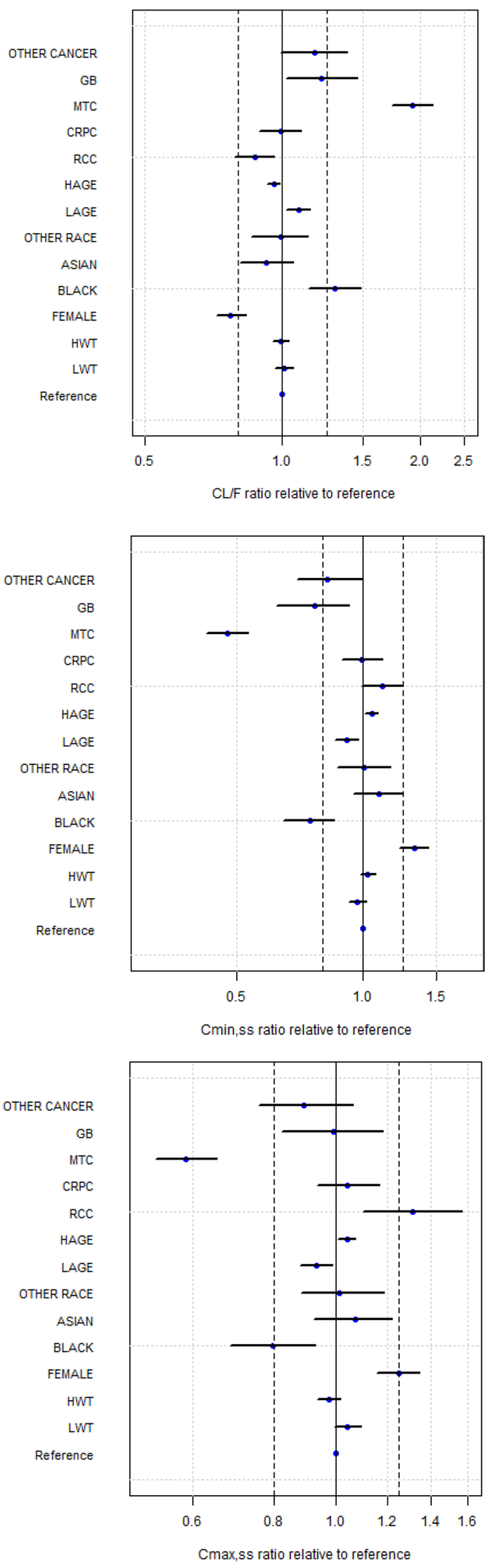
of efflux transporter MRP2 [25], so concomitant administration of an MRP2 inducer could potentially increase cabozantinib clearance by enhancing hepatic and/or intestinal drug MRP2-mediated transport activity. Although overall use of concomitant MRP2 inducers was not documented for MTC patients in study XL184-301, only 5.5\% of MTC subjects (12 of 219) administered cabozantinib were reported to have received MRP2 inducer (and moderate CYP3A4 inducer) dexamethasone.

Cabozantinib plasma clearance $(\mathrm{CL} / F)$ may also appear to be higher if oral bioavailability $(F)$ decreased with increasing cabozantinib dose. The approved cabozantinib dose for MTC patients (140-mg) is higher than the dose approved for RCC patients and dose generally administered to non-MTC patient populations $(60-\mathrm{mg})$, and steady-state $\mathrm{CL} / F$ in the MTC popPK analysis $(4.4 \mathrm{~L} / \mathrm{h})$ was higher than that determined in the RCC popPK analysis $(2.2 \mathrm{~L} / \mathrm{h})$. However, no decrease in cabozantinib oral bioavailability was evident in a cross-study analysis indicating generally dose-linear PK for tablet and capsule formulations over a broad dose range (20-140 mg) [25]. In addition, lower cabozantinib exposures associated with higher relative $\mathrm{CL} / F$ in MTC patients were only observed at steady state (day 29) and not at day 1.

Alternatively, estimates of CL/ $F$ values from MTC subjects that tolerate a $140-\mathrm{mg}$ daily cabozantinib dose may be higher than the overall study population if they reflect a sub-population that tolerates this higher dose at steady state based on a faster relative intrinsic clearance. In the MTC popPK analysis, high drop-out rate or early discontinuation was also considered to possibly explain the lower day 29 concentrations in MTC patients relative to HVs [11]. If subjects with low clearance and higher exposures dropped out or discontinued the study early due to treatment-emergent AEs, only those subjects with higher clearances resulting in lower, more tolerable exposures would remain. This scenario is unlikely considering $79 \%$ of the patients in the MTC popPK analysis contributed PK samples on both days 1 and 29.

Finally, a more detailed PK sampling of the terminal elimination phase was included in the RCC popPK analysis (up to 504-h post-dose in HVs) than in the MTC popPK analysis where the final PK sample was taken at approximately 24 -h post-dose. As cabozantinib has a relatively long plasma terminal half-life (HV mean: $118 \mathrm{~h}$ [25]), plasma clearance could have been underestimated in the MTC popPK analysis based on a more limited PK data collection of terminal elimination phase. However, the integrated popPK model developed subsequently included exposure data from patients with different tumor types and HVs; the addition of all covariates, including demographic (age, weight sex, and race) and population (RCC, CRPC, MTC, GB, and advanced malignancies) on both $\mathrm{CL} / F$ and $\mathrm{Vc} / F$ resulted in an adequate fit to the data. The magnitude of most demographic and population-specific covariate effects on cabozantinib PK was small, except for MTC patient population who had a substantially larger estimated cabozantinib CL/F. Thus, model-related and PK sampling differences do not appear to underlie the higher $\mathrm{CL} / F$ values in MTC patients evident at steady state.

\section{Conclusion}

In summary, results from the integrated popPK analysis indicate that compared with other cancer patient groups (RCC, CRPC, and GB), MTC patients clear cabozantinib faster and thus have lower dose-normalized steady-state plasma exposures. Cabozantinib PK appears to be timevarying in MTC patients, as day $1 \mathrm{CL} / F$ values were lower and comparable to those in non-MTC cancer patient populations. Several possible factors may underlie the higher cabozantinib clearance observed in MTC patients; however, an exact cause has yet to be identified. Based on the integrated popPK analysis, non-MTC cancer patient cohorts (including RCC patients) appear to have comparable cabozantinib clearance to that of HVs.

Acknowledgements The authors wish to respectfully thank the medical professionals and patients that participated in the clinical trial of cabozantinib reported in this manuscript. The authors also wish to acknowledge Susan K. Paulson, Ph.D., for her assistance in the preparation of this manuscript. This assistance was fully funded by Exelixis, Inc.

Funding The studies described in this manuscript were supported by Exelixis, Inc.

\section{Compliance with ethical standards}

Conflict of interest Steven Lacy and Linh Nguyen are stockholders and current employees of Exelixis, Inc. Dale Miles was an employee of Exelixis, Inc. when this work was performed, and is currently employed at Genentech, Inc. Jace Nielsen, Bei Yang, and Matt Hutmacher are employees of Ann Arbor Pharmacometrics Group (A2PG), Inc. who designed and conducted the popPK modeling reported in this manuscript and was funded by Exelixis, Inc. Bei Yang is currently employed by Luoxin Biotechnology (Shanghai) Co., Ltd. The authors contributed significantly to the design, conduct, analyses, and interpretation of the data, and were involved in the preparation, review, and approval of this manuscript.

Ethical approval The clinical studies were conducted in accordance with the World Medical Association Declaration of Helsinki, the International Conference on Harmonisation Tripartite Guideline for Good Clinical Practice, and all applicable local regulations. Study protocols and informed consent documents were reviewed and approved by the Institutional Review Board (IRB) of participating institutions, and informed consent was obtained from all participants before any studyspecified procedures were undertaken. 
Open Access This article is distributed under the terms of the Creative Commons Attribution 4.0 International License (http://creativeco mmons.org/licenses/by/4.0/), which permits unrestricted use, distribution, and reproduction in any medium, provided you give appropriate credit to the original author(s) and the source, provide a link to the Creative Commons license, and indicate if changes were made.

\section{References}

1. Yakes FM, Chen J, Tan J et al (2011) Cabozantinib (XL184), a novel MET and VEGFR2 inhibitor, simultaneously suppresses metastasis, angiogenesis, and tumor growth. Mol Cancer Ther 10(12):2298-2308

2. Cometriq ${ }^{\circledR}$ (cabozantinib) capsules (2012) US prescribing information. Exelixis Inc., South San Francisco. https://www. accessdata.fda.gov/drugsatfda_docs/label/2012/203756lbl.pdf. Accessed 05 Apr 2018

3. Cometriq ${ }^{\circledR}$ (cabozantinib) capsules (2016) Summary of product characteristics. Ipsen Pharma, Boulogne-Billancourt, France. https://www.medicines.org.uk/emc/product/4408. Accessed 05 Apr 2018

4. Cabometyx ${ }^{\mathrm{TM}}$ (cabozantinib) tablets (2016) US prescribing information Exelixis Inc., South San Francisco. https://www.acces sdata.fda.gov/drugsatfda_docs/label/2016/208692s0001bl.pdf. Accessed 05 Apr 2018

5. Cabometyx ${ }^{\text {TM }}$ (cabozantinib) tablets (2016) Summary of product characteristics. Ipsen Pharma, Boulogne-Billancourt, France. https://www.medicines.org.uk/emc/product/4331. Accessed 05 Apr 2018

6. Study of Cabozantinib (XL184) vs Placebo in Subjects With Hepatocellular Carcinoma Who Have Received Prior Sorafenib. ClinicalTrials.gov Identifier NCT01908426. https://clinicaltrials. gov/ct2/show/NCT01908426?term=cabozantinib\&rank $=10$

7. Nguyen L, Benrimoh N, Xie Y et al (2016) Pharmacokinetics of cabozantinib tablet and capsule formulations in healthy adult subjects. Anticancer Drugs 27(7):669-678

8. Kurzock R, Sherman SI, Ball DW et al (2011) Activity of XL184 (cabozantinib), an oral tyrosine kinase inhibitor, in patients with medullary thyroid cancer. J Clin Oncol 29(19):2660-2666

9. Elisei R, Schlumberger MJ, Müller SP et al (2013) Cabozantinib in progressive medullary cancer. J Clin Oncol 31(29):3639-3646

10. EMA (2013) European Public Assessment Report (EPAR) for Cometriq. http://www.ema.europa.eu/docs/en_GB/docum ent_library/EPAR_-_Public_assessment_report/human/002640/ WC500163705.pdf. Accessed 18 Oct 2017

11. Miles D, Jumbe S, Lacy S et al (2016) Population pharmacokinetic model of cabozantinib in patients with medullary thyroid carcinoma and its application to an exposure-response analysis. Clin Pharmacokinet 55(1):93-105

12. Miles DR, Wada DR, Jumbe NL et al (2016) Population pharmacokinetic/pharmacodynamic modeling of tumor growth kinetics in medullary thyroid cancer patients receiving cabozantinib. Anticancer Drugs 27(4):328-341

13. Choueiri TK, Pal SK, McDermott DF et al (2014) A Phase 1 study of cabozantinib (XL184) in patients with renal cell carcinoma. Ann Oncol 25(8):1603-1608
14. Choueiri TK, Escudier B, Powles T et al (2016) Cabozantinib versus everolimus in advanced renal cell carcinoma (METEOR): final results from a randomized, open-label phase 3 trial. Lancet Oncol 17(7):917-927

15. Smith M, De Bono J, Stemberg C et al (2016) Phase III study of cabozantinib in previously treated metastatic castration-resistant prostate Cancer: COMET-1. J Clin Oncol 34(25):3005-3013

16. Singh H, Brave M, Beaver JA et al (2016) U.S. food and drug administration approval: cabozantinib for treatment of advanced renal cell carcinoma. Clin Cancer Res 23(2):330-335

17. European Medicines Agency (2016) European Public Assessment Report (EPAR) for Cabometyx. http://www.ema.europa.eu/docs/ en_GB/document_library/EPAR_-_Public_assessment_report/ human/004163/WC500214070.pdf. Accessed 18 Oct 2017

18. Schiff D, Desjardins A, Cloughesy T et al (2016) Phase 1 dose escalation trial of the safety and pharmacokinetics of cabozantinib concurrent with temozoloide and radiotherapy or temozolomide after radiotherapy in newly diagnosed patients with high-grade gliomas. Cancer 122(4):582-587

19. Smith MR, Sweeney CJ, Corn PG et al (2014) Cabozantinib in chemotherapy-pretreated metastatic castration-resistant prostate cancer: results of a phase II nonrandomized expansion study. J Clin Oncol 32(30):3391-3399

20. Smith DC, Smith MR, Sweeney C et al (2013) Cabozantinib in patients with advanced prostate cancer: results of a phase II randomized discontinuation trial. J Clin Oncol 31(4):412-419

21. Lacy S, Hsu B, Miles D et al (2015) Metabolism and disposition of cabozantinib in healthy male volunteers and pharmacologic characterization of its major metabolites. Drug Metab Dispos 43:1190-1207

22. Kowalski KG, Hutmacher MM (2014) Covariate selection in pharmacometric analyses: a review of methods. Br J Clin Pharmacol 79:132-147

23. Gastonguay M (2011) Full covariate models as an alternative to methods relying on statistical significance for inferences about covariate effects: a review of methodology and 42 case studies. PAGE 20:Abstr 2229

24. Yano Y, Beal SL, Scheiner LB (2001) Evaluating pharmacokinetic/pharmacodynamics models using the posterior predictive check. J Pharmacokinet Pharmacodyn 28:171-92

25. Lacy S, Miles D, Nguyen L (2017) Clinical pharmacokinetics and pharmacodynamics of cabozantinib. Clin Pharmacokinet $56: 477-491$

26. Lu J-F, Claret L, Sutjandra L et al (2010) Population pharmacokinetic/pharmacodynamics modeling for the time course of tumor shrinkage by motesanib in thyroid cancer patients. Cancer Chemother Pharmacol 66:1151-1158

27. Wang Z, Schuetz EG, Xu Y et al (2013) Interplay between vitamin $\mathrm{D}$ and drug metabolizing enzyme CYP3A4. J Steroid Biochem Mol Biol 136:54-58

28. Drocourt L, Ourlin J-C, Pascussi J-M et al (2002) Expression of CYP3A4, CYP2B6 and CYP2C9 is regulated by the vitamin D receptor pathway in primary human hepatocytes. J Biol Chem 277:25125-25132

29. Nguyen L, Holland J, Miles D et al (2015) Pharmacokinetic (PK) drug interaction studies of cabozantinib: effect of CYP3A4 inducer rifampin and inhibitor ketoconazole on cabozantinib plasma PK, and effect of cabozantinib on CYP2C8 probe substrate rosiglitazone plasma PK. J Clin Pharmacol 55(9):1012-1023 